\title{
Investigation of Solid-state Transformations Under Extreme Thermal Transients Using In-situ TEM Heating Experiments
}

Sriram Vijayan ${ }^{1}$, Meiyue Shao ${ }^{1}$, Rongxuan Wang ${ }^{2}$, Zhenyu Kong ${ }^{2}$ and Joerg Jinschek ${ }^{1}$

${ }^{1}$ The Ohio State University, Columbus, Ohio, United States, ${ }^{2}$ Virginia Polytechnic Institute and State University, Blacksburg, Virginia, United States

Micro-electro-mechanical systems (MEMS) based heating stages have revolutionized in-situ transmission electron microscopy (TEM) experiments and are widely used to observe thermally activated processes at high spatial resolution [1]. TEM heating experiments are routinely performed to observe phenomena such as sublimation and melting of metal nanoparticles, phase transformations in complex alloys, and dewetting of thin films, to name a few. These MEMS-based micro-heaters are ideal to observe solid-state transformations in materials at the atomic scale, because of their reduced sample drift, small thermal mass, fast heater response times, and high temperature homogeneity across the heater surface $[2,3]$.

In metal additive manufacturing (AM) processes, an electron (EBM) or laser heat source melts powder particles/wires that are deposited in a 'layer-by-layer' process to build an AM part [4]. The most recent deposited layer experiences rapid melting \& solidification, while previously deposited layers undergo complex and repeated thermal cycling processes with rapid (re)heating and (re)cooling causing intricate alloy phase transformations. The unidirectional heat flow and complex thermal cycles results in AM parts with anisotropic microstructures that consists of columnar grains growing along the build direction. Nonuniform properties resulting from these anisotropic microstructures have limited the qualification of AM parts for critical load bearing applications. Major efforts are being directed towards developing AM parts with equiaxed microstructures. However, these efforts are limited by the lack of understanding on the mechanisms that govern solid-to-solid transformations in AM-like processes [5]. AM parts experience large thermal gradients $\left(10^{4}-10^{7} \mathrm{~K} / \mathrm{m}\right)$ and high cooling rates $\left(10^{3}-10^{5} \mathrm{~K} / \mathrm{s}\right)$ that are drastically different to parameters in conventional manufacturing processes. In order to understand the mechanisms that govern these solid state transformations in AM under extreme thermal transients, we demonstrate the use of a new in-situ MEMS based micro-heater that can simulate AM process conditions inside a TEM.

In this report, we describe the methodology to generate a thermal gradient across a TEM sample using a MEMS-based heater. Subsequently, this device is used to investigate the influence of thermal gradients on solid-solid interfaces in EBM AM Ti-6Al-4V (Ti64). A commercially available MEMS-based micro heater (DENS solutions) is modified using a dual beam focused ion beam scanning electron microscope, Helios-600, Thermo Fisher Scientific (FIB-SEM). A window with dimensions $(25 \mu \mathrm{m}$ X $30 \mu \mathrm{m})$ is milled through the silicon nitride membrane next to the metallic spiral heating element in the FIB (Figure 1(a)). Infra-red thermal camera was used to measure the temperature distribution across the MEMS heater surface (Figure 1(b)). The thermal gradient (dT/dx) was measured from the heater to the edge of the window and found to be $\sim 10^{6} \mathrm{~K} / \mathrm{m}$, which is within the range of thermal gradients observed in AM processes. The FIB-cut lamella obtained from a Ti64 gas atomized powder particle was placed [6] across the modified window with one end on the heater and the other end on the silicon nitride membrane that acts as the sink (Figure 2(a-c)). Finally, in situ TEM heating experiments were performed to study solidsolid interface stability under AM like conditions [7]. 

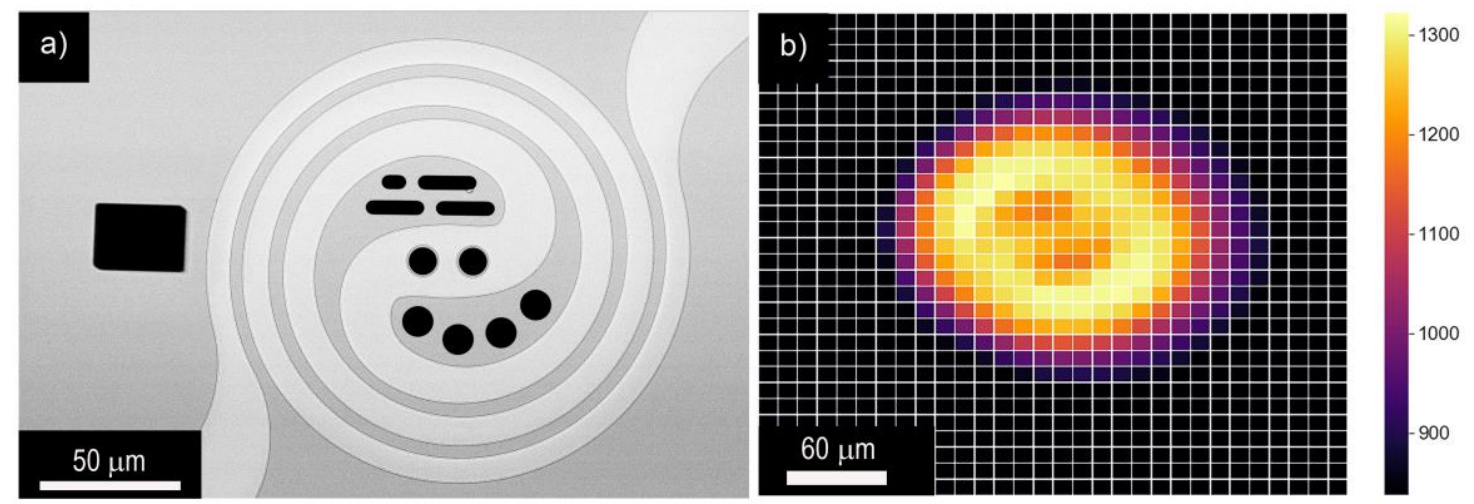

Figure 1. (a) MEMS micro heater with a modified window next to the spiral heating element, (b) Infrared thermal image of the temperature distribution across the MEMS microheater device at $1300^{\circ} \mathrm{C}$
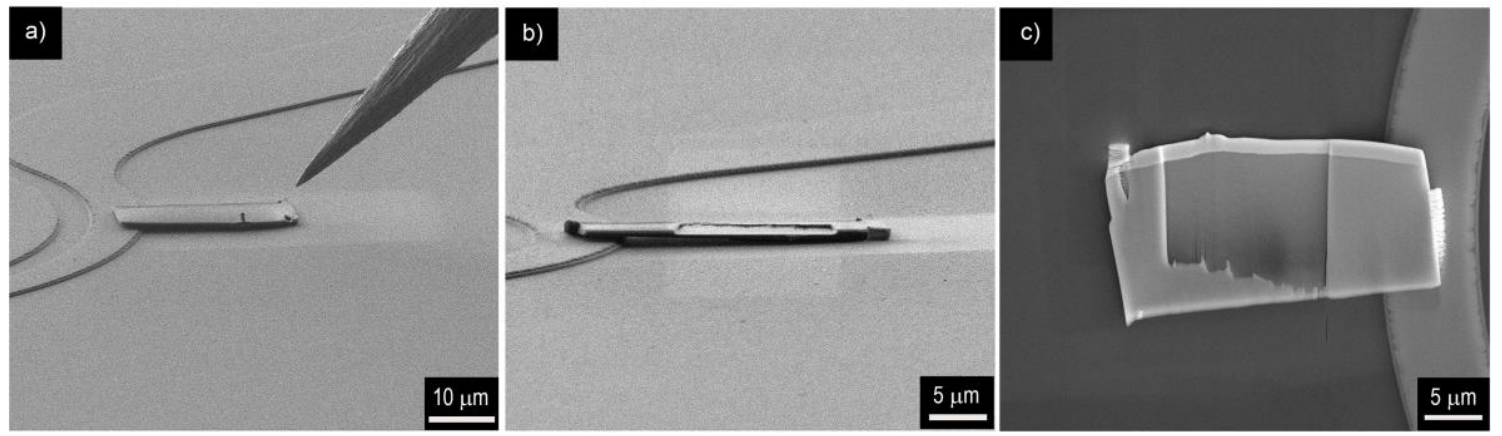

Figure 2. Secondary ion beam (a-b) and secondary electron (c) SEM image of a Ti64 TEM lamella placed over the modified window of the MEMS micro heater. The right side of the lamella is attached to the heating element (heater) and the left end is attached to the silicon nitride membrane (sink).

\section{References}

[1] Jungjohann, K. \& Carter, C.B. (2016). In situ and operando. In Transmission Electron Microscopy: Diffraction Imaging and Spectrometry, Carter, C.B. \& Williams, D.B. (Eds.), p. 17-80.

[2] L. Mele, et al., Microscopy Research and Technique 79 (2016) p. 239.

[3] Vijayan, S. et al, Studies of thermally activated processes in gas atomized Al alloy powders: in situ STEM heating experiments on FIB-cut cross sections, J. Mater. Sci., 54 (2019), p 9921-9932.

[4] Liu, S., Shin, Y.C., Additive manufacturing of Ti6Al4V alloy: a review, Mater. \& Des., 164 (2019), p. 107552:1-23.

[5] Kirka, M. et al, Solidification and solid-state sciences in metals additive manufacturing, Scr. Mat., 135 (2017) p. 130-134.

[6] Vijayan et al, Focused ion beam preparation of specimens for micro-electro-mechanical system-based transmission electron microscopy heating experiments, Microsc. \& Microanal., 23(2017) p.708-716.

[7] The research is sponsored by the Department of the Navy, Office of Naval Research under ONR award number N00014-18-1-2794. Any opinions, findings, and conclusions or recommendations expressed in this material are those of the author(s) and do not necessarily reflect the views of the Office of Naval Research. Electron microscopy was performed at the Center for Electron Microscopy and Analysis (CEMAS) at The Ohio State University. 